\title{
THE PRODUCT OF INDEPENDENT RANDOM VARIABLES WITH SLOWLY VARYING TRUNCATED MOMENTS
}

\author{
TAKAAKI SHIMURA
}

(Received 30 October 1995; revised 1 April 1996)

Communicated by A. G. Pakes

\begin{abstract}
The Mellin-Stieltjes convolution and related decomposition of distributions in $\boldsymbol{M}(\boldsymbol{\alpha})$ (the class of distributions $\mu$ on $[0, \infty)$ with slowly varying $\alpha$ th truncated moments $\left.\int_{0}^{x} t^{\alpha} \mu(d t)\right)$ are investigated. Maller shows that if $X$ and $Y$ are independent non-negative random variables with distributions $\mu$ and $\nu$, respectively, and both $\mu$ and $\nu$ are in $D_{2}$, the domain attraction of Gaussian distribution, then the distribution of the product $X Y$ (that is, the Mellin- Stieltjes convolution $\mu \circ v$ of $\mu$ and $\nu$ ) also belongs to it. He conjectures that, conversely, if $\mu \circ \nu$ belongs to $D_{2}$, then both $\mu$ and $\nu$ are in it. It is shown that this conjecture is not true: there exist distributions $\mu \in D_{2}$ and $v \notin D_{2}$ such that $\mu \circ v$ belongs to $D_{2}$. Some subclasses of $D_{2}$ are given with the property that if $\mu \circ \nu$ belongs to it, then both $\mu$ and $\nu$ are in $D_{2}$.
\end{abstract}

1991 Mathematics subject classification (Amer. Math. Soc.): primary 60E05; secondary 60E07, 60F05. Keywords and phrases: domain of attraction; truncated moment; slowly varying function; regular variation; Mellin-Stieltjes convolution.

\section{Introduction}

Let $X$ and $Y$ be independent positive random variables with distributions $\mu$ and $\nu$, respectively. We denote the distribution of the product $X Y$ by $\mu \circ v$ and call it the Mellin-Stieltjes convolution (MS- convolution) of $\mu$ and $\nu$. A distribution $\mu_{1}$ is said to be a factor of a distribution $\mu$, if $\mu=\mu_{1} \circ v$ with some $v$. Let $\boldsymbol{M}(\alpha)(\alpha>0)$ be the class of distributions $\mu$ on $[0, \infty)$ whose $\alpha$ th truncated moments $\int_{0}^{x} t^{\alpha} \mu(d t)$ are slowly varying. The purpose of this paper is to study properties of distributions in $M(\alpha)$ related to MS-convolution. Let $D_{2}$ be the domain of attraction of Gaussian distribution. Maller [5] shows that if $X$ and $Y$ are independent random variables both with distributions in $D_{2}$, then the distribution of the product $X Y$ also belongs to it. In the converse direction, he shows that if a distribution of product of two independent

(C) 1997 Australian Mathematical Society $0263-6115 / 97 \$ A 2.00+0.00$ 
random variables belongs to $D_{2}$ and one of them has finite variance, then the other is in $\boldsymbol{D}_{2}$. Furthermore, he conjectures that finite variance condition could be weakened to being in $D_{2}$. Since $D_{2}$ is identical with the class of distributions $\mu$ with slowly varying truncated variances $\int_{|t|<x} t^{2} \mu(d t)$, these facts mean that $\boldsymbol{M}(2)$ is closed under MS-convolution, and that, if one factor of a distribution in $\boldsymbol{D}_{2}$ has finite variance, then the other belongs to $\boldsymbol{D}_{2}$. We deal with this problem in detail. Considering the relation between the truncated moments of two distributions and that of their MS-convolution, we give some conditions for each factor of $\mu \circ v$ to belong to $M(\alpha)$. The general results on the decomposition of non-decreasing slowly varying functions are applicable. In the end of this paper, we construct a counter-example for Maller's conjecture: there exists a distribution $\mu \notin D_{2}$ such that the MS-convolution of $\mu$ and $\nu$ belongs to $D_{2}$ for every $v$ in $D_{2}$ with infinite variance.

\section{Preliminaries}

We prepare some notations and fundamental facts, which are in Bingham et al. [1], Feller [2], Gnedenko and Kolmogorov [3], Seneta [6] and Shimura [7, 8]. The totality of all probability measures on non-negative numbers $[0, \infty)$ is denoted by $\boldsymbol{P}$. Through this paper, we extend MS-convolution to the all distributions in $\boldsymbol{P}$ since the mass on 0 is not essential. A positive measurable function $f$ is said to be slowly varying (s.v.) if $\lim _{x \rightarrow \infty} f(k x) / f(x)=1$ for each $k>0$. If $f$ is monotone, this is equivalent to $\lim _{x \rightarrow \infty} f(2 x) / f(x)=1$. Slowly varying functions have the following representation: A function $f$ defined on $[A, \infty), A>0$, is s.v. if and only if there exists a positive number $B \geq A$ satisfying for all $x \geq B$ we have $f(x)=$ $c(x) \exp \left(\int_{B}^{x} \varepsilon(t) t^{-1} d t\right)$, where $c(x)$ is a bounded positive measurable function on $[B, \infty)$ satisfying $\lim _{x \rightarrow \infty} c(x)=c(0<c<\infty)$, and $\varepsilon(t)$ is a continuous function on $[B, \infty)$ satisfying $\lim _{t \rightarrow \infty} \varepsilon(t)=0$. This representation leads to the following lemma.

LEMMA 2.1. If $l$ is s.v., then for some $B>0,(l(x) x) /(l(y) y)$ is bounded with respect to $x$ and $y$ satisfying $x \leq y$ and $y \geq B$.

We say that non-negative non-decreasing $f$ is decomposed into components $f_{1}$ and $f_{2}$, if both $f_{1}$ and $f_{2}$ are non-negative non-decreasing and $f=f_{1}+f_{2}$. Concerning the decomposition of non-decreasing s.v. functions, the following are known. A non-negative non-decreasing function $f$ is said to be dominatedly non-decreasing if $\lim \sup _{x \rightarrow \infty}(f(2 x)-f(x))<\infty$. Then $f$ is s.v. and the class of dominatedly nondecreasing functions is closed under sum and decomposition. On the decomposition of non-decreasing s.v. functions, we recall the following in Shimura [7]. Related facts concerning monotone regularly varying functions are given in Shimura [8]. 
THEOREM 2.2. (1) Every non-zero component of $f$ is s.v. if and only if $f$ is dominatedly non-decreasing. In this case, every non-zero component is dominatedly non-decreasing.

(2) A component $f_{1}$ of a non-decreasing s.v. $f$ satisfying $\liminf _{\rightarrow \infty} f_{1}(x) / f(x)>0$ is s.v.

(3) For any unbounded non-decreasing s.v. function $f$, there exists a non-decreasing function $\tilde{f}$ that is asymptotically equal to $f$ but not dominatedly non-decreasing.

(4) For a dominatedly non-decreasing $f$, $\lim \sup _{x \rightarrow \infty} f(x) / \log x<\infty$.

Let $\boldsymbol{F}(\alpha), \boldsymbol{S}(\alpha)$, and $\boldsymbol{C}(\alpha)(\alpha>0)$ denote the subclasses of $\boldsymbol{P}$ defined by the following conditions: $\mu$ is in $F(\alpha)$ if $\mu$ has dominatedly non-decreasing $\alpha$ th truncated moment, $\mu$ is in $S(\alpha)$ if $\lim _{x \rightarrow \infty} \int_{0}^{x^{2}} t^{\alpha} \mu(d t) / \int_{0}^{x} t^{\alpha} \mu(d t)=1 ; \mu$ is in $C(\alpha)$ if $\lim \sup _{x \rightarrow \infty} \int_{0}^{x^{2}} t^{\alpha} \mu(d t) / \int_{0}^{x} t^{\alpha} \mu(d t)<\infty$. It is easy to see that $\mu$ is in $S(\alpha)$ if and only if its truncated $\alpha$ th moment is written as $\int_{0}^{x} t^{\alpha} \mu(d t)=l(\log x)$ for some non-decreasing s.v. function $l$. Similarly, $\mu$ is in $\boldsymbol{C}(\alpha)$ if and only if $\int_{0}^{x} t^{\alpha} \mu(d t)=$ $\exp f(\log x)$ with some dominatedly non-decreasing function $f$. Although $S(\alpha)$ is a subclass of $\boldsymbol{M}(\alpha)$ and $\boldsymbol{C}(\alpha)$, it is not a subclass of $\boldsymbol{F}(\alpha)$ (Theorem $2.2(3)$ ). $\boldsymbol{C}(\alpha)$ is not a subclass of $M(\alpha)$ as we shall show in Section 4.

Let $X_{1}, X_{2}, \ldots$ be $R^{1}$-valued i.i.d. (independent and identically distributed) random variables with distribution $\nu$. If, for suitably chosen constants $B_{n}>0$ and $A_{n} \in R^{1}$, the distribution of $B_{n}^{-1} \sum_{k=1}^{n} X_{k}-A_{n}$ converges to a distribution $\mu$ as $n \rightarrow \infty$, then we say that $\nu$ is attracted to $\mu$. The totality of distributions attracted to $\mu$ is called the domain of attraction of $\mu$. We denote the domain of attraction of Gaussian distribution by $\boldsymbol{D}_{2}$. If, for suitably chosen constants $B_{n}>0$, the distribution of $B_{n}^{-1} \sum_{k=1}^{n} X_{k}$ converges to 1 in probability as $n \rightarrow \infty$, then we say that $v$ is relatively stable. Those classes are characterized by truncated moments as follows: $v$ belongs to $D_{2}$ if and only if $v$ has s.v. truncated variance $\int_{|t|<x} t^{2} v(d t)$. Under the assumption that $v$ is in $P, v$ is relatively stable if and only if $\nu$ belongs to $M(1)$.

\section{Mellin-Stieltjes convolution of slow varying truncated moments}

As we mentioned, Maller shows that $\boldsymbol{M}(2)$ is closed under MS-convolution. By change of variables, this implies that, for each $\alpha>0, M(\alpha)$ is closed. We give a new proof of this fact and investigate the relationship between the growth order of the truncated moment of MS-convolution and that of its factors.

THEOREM 3.1. If $\mu$ is in $M(\alpha)$, then

$$
\lim _{x \rightarrow \infty} \int_{0}^{x} t^{\alpha} \mu \circ v(d t) / \int_{0}^{x} t^{\alpha} \mu(d t)=\int_{0}^{\infty} t^{\alpha} \nu(d t)
$$


If, moreover, $v$ has finite $\alpha$ th moment, then $\mu \circ v$ belongs to $M(\alpha)$.

Proof. Assume that $\int_{0}^{\infty} t^{\alpha} v(d t)$ is finite. Since $\int_{0}^{x} t^{\alpha} \mu \circ v(d t)=\int_{0}^{\infty} t^{\alpha} v(d t)$ $\int_{0}^{x / t} s^{\alpha} \mu(d s)$, we have

$$
\begin{aligned}
\frac{\int_{0}^{x} t^{\alpha} \mu \circ \nu(d t)}{\int_{0}^{x} s^{\alpha} \mu(d s)} & =\int_{0}^{\infty} t^{\alpha} \nu(d t) \frac{\int_{0}^{x / t} s^{\alpha} \mu(d s)}{\int_{0}^{x} s^{\alpha} \mu(d s)} \\
& =\int_{0}^{1} v(d t) \frac{x^{\alpha} \int_{0}^{x / t} s^{\alpha} \mu(d s)}{(x / t)^{\alpha} \int_{0}^{x} s^{\alpha} \mu(d s)}+\int_{1}^{\infty} t^{\alpha} v(d t) \frac{\int_{0}^{x / t} s^{\alpha} \mu(d s)}{\int_{0}^{x} s^{\alpha} \mu(d s)} .
\end{aligned}
$$

Since $\sup _{x \geq B} \sup _{t \in(0.1]}\left(x^{\alpha} \int_{0}^{x / t} s^{\alpha} \mu(d s)\right) /\left((x / t)^{\alpha} \int_{0}^{x} s^{\alpha} \mu(d s)\right)<\infty$ by Lemma 2.1, the first term goes to $\int_{0}^{1} t^{\alpha} v(d t)$ as $x \rightarrow \infty$ by the bounded convergence theorem. The second term converges to $\int_{1}^{\infty} t^{\alpha} \nu(d t)$ because $\int_{0}^{x / t} s^{\alpha} \mu(d s) / \int_{0}^{x} s^{\alpha} \mu(d s) \leq 1$ on $t \in[1, \infty)$. If $\int_{0}^{\infty} t^{\alpha} v(d t)$ is infinite, then, by Fatou's lemma

$$
\liminf _{x \rightarrow \infty} \int_{0}^{x} t^{\alpha} \mu \circ v(d t) / \int_{0}^{x} t^{\alpha} \mu(d t) \geq \int_{0}^{\infty} t^{\alpha} v(d t)=\infty .
$$

THEOREM 3.2 (Maller [5, Theorem 1], if $\alpha=2) . M(\alpha)(\alpha>0)$ is closed under MS-convolution.

PROOF. Notice that

$$
\begin{aligned}
\frac{\int_{x}^{2 x} t^{\alpha} \mu \circ v(d t)}{\int_{0}^{x} t^{\alpha} \mu \circ v(d t)} & \leq \frac{\int_{0}^{\sqrt{2 x}} t^{\alpha} \nu(d t) \int_{x / t}^{2 x / t} s^{\alpha} \mu(d s)}{\int_{0}^{\sqrt{2 x}} t^{\alpha} \nu(d t) \int_{0}^{x / t} s^{\alpha} \mu(d s)}+\frac{\int_{0}^{\sqrt{2 x}} t^{\alpha} \mu(d t) \int_{x / t}^{2 x / t} s^{\alpha} v(d s)}{\int_{0}^{\sqrt{2 x}} t^{\alpha} \mu(d t) \int_{0}^{x / t} s^{\alpha} \nu(d s)} \\
& \leq \sup _{t<\sqrt{2 x}} \frac{\int_{x / t}^{2 x / t} s^{\alpha} \mu(d s)}{\int_{0}^{x / t} s^{\alpha} \mu(d s)}+\sup _{t<\sqrt{2 x}} \frac{\int_{x / t}^{2 x / t} s^{\alpha} v(d s)}{\int_{0}^{x / t} s^{\alpha} \nu(d s)} .
\end{aligned}
$$

Since $\mu$ belongs to $M(\alpha)$, the first term tends to 0 as $x \rightarrow \infty$. Similarly, the second term goes to 0 . Thus we get $\lim _{x \rightarrow \infty} \int_{x}^{2 x} t^{\alpha} \mu \circ v(d t) / \int_{0}^{x} t^{\alpha} \mu \circ v(d t)=0$, which means that $\mu \circ \nu$ belongs to $M(\alpha)$.

By the above theorems, we obtain the following result.

COROLlaRY 3.3. If $\mu$ is in $M(\alpha)$ and $\nu$ is in $M(\beta)$ with $\alpha \leq \beta$, then $\mu \circ v$ belongs to $M(\alpha)$.

In the above corollary, if $\alpha<\beta$, then the growth order of the truncated moment of $\mu \circ \nu$ is given by (3.1). We will compare in the next section the truncated moment of $\mu \circ \nu$ and the product of the truncated moments of the two factors, including the case $\alpha=\beta$. 


\section{Decomposition problem of distributions in $M(\alpha)$}

In this section, we investigate properties of factors of distributions in $\boldsymbol{M}(\alpha)$. One purpose is to give some conditions for every factor to belong to $M(\alpha)$. In particular, $\boldsymbol{S}(\alpha)$ is closed under MS-convolution and decomposition. Another is to prove that Maller's conjecture is not true. Namely, we will prove that if $v$ is in $C(\alpha)$, then $\mu \circ \nu$ belongs to $\boldsymbol{M}(\alpha)$ for every $\boldsymbol{\mu}$ in $\boldsymbol{M}(\alpha)$ with infinite $\alpha$ th moment. First, applying Theorem 2.2, we give a theorem on the decomposition problem.

THEOREM 4.1. Every factor of distribution in $\boldsymbol{F}(\alpha)$ belongs to $\boldsymbol{F}(\alpha)$.

PROOF. We assume that $\mu \circ \nu$ has dominatedly non-decreasing $\alpha$ th truncated moment and $v(1, \infty)>0$ without loss of generality. Notice that

$$
\int_{0}^{x} t^{\alpha} \mu \circ \nu(d t)=\int_{0}^{\infty} t^{\alpha} v(d t) \int_{0}^{x / t} s^{\alpha} \mu(d s)=\sum_{k=1}^{\infty} \int_{k-1}^{k} t^{\alpha} \nu(d t) \int_{0}^{x / t} s^{\alpha} \mu(d s) .
$$

Set $v_{k}(x)=\int_{k-1}^{k} \int_{0}^{x / t} s^{\alpha} \mu(d s) t^{\alpha} \nu(d t)$. Then $\int_{0}^{x} t^{\alpha} \mu \circ \nu(d t)=\sum_{k=1}^{\infty} v_{k}(x)$. Choose and fix an integer $k \geq 2$ such that $v_{k}$ is not identically zero. By Theorem $2.2(1), v_{k}$ is dominatedly non-decreasing. Since

$$
\int_{k-1}^{k} t^{\alpha} \nu(d t) \int_{0}^{x / k} s^{\alpha} \mu(d s) \leq v_{k}(x) \leq \int_{k-1}^{k} t^{\alpha} \nu(d t) \int_{0}^{x /(k-1)} s^{\alpha} \mu(d s),
$$

we have

$$
\int_{x}^{2 x} s^{\alpha} \mu(d s) \leq\left(\int_{k-1}^{k} t^{\alpha} \nu(d t)\right)^{-1}\left(v_{k}(2 k x)-v_{k}((k-1) x)\right) .
$$

By the dominated non-decrease of $v_{k}$, we get $\lim \sup _{x \rightarrow \infty} \int_{x}^{2 x} s^{\alpha} \mu(d s)<\infty$. Hence $\mu \in \boldsymbol{F}(\alpha)$. So is $\nu$.

REMARK. In this proof, we get the dominated non-decrease of the truncated moment of $\mu$ from the dominated non-decrease of $v_{k}$. Similarly, if it is shown that $v_{k}$ is s.v., then we can prove that $\mu$ belongs to $\boldsymbol{M}(\alpha)$ by (4.1). But, it is impossible to show that $v_{k}$ is s.v. under the assumption that $\int_{0}^{x} t^{\alpha} \mu \circ v(d t)=\sum_{k=1}^{\infty} v_{k}(x)$ is s.v., as will be shown in Section 4.

LEMMA 4.2. If $\mu \circ v$ is in $M(\alpha)$, then

$$
\limsup _{x \rightarrow \infty} \int_{0}^{x} t^{\alpha} \mu(d t) / \int_{0}^{x} t^{\alpha} \mu \circ v(d t) \leq\left(\int_{0}^{\infty} t^{\alpha} \nu(d t)\right)^{-1} .
$$


PROOF. For arbitrary $k>0$,

$$
\begin{aligned}
\int_{0}^{k x} t^{\alpha} \mu \circ v(d t) & \geq \int_{0}^{k} t^{\alpha} \nu(d t) \int_{0}^{k x / t} s^{\alpha} \mu(d s) \\
& \geq \int_{0}^{k} t^{\alpha} \nu(d t) \int_{0}^{x} s^{\alpha} \mu(d s) .
\end{aligned}
$$

Since $\int_{0}^{x} t^{\alpha} \mu \circ v(d t)$ is s.v., we get

$$
\limsup _{x \rightarrow \infty} \int_{0}^{x} t^{\alpha} \mu(d t) / \int_{0}^{x} t^{\alpha} \mu \circ \nu(d t) \leq\left(\int_{0}^{k} t^{\alpha} \nu(d t)\right)^{-1} .
$$

Letting $k \rightarrow \infty$, we get the conclusion.

LEMMA 4.3. If $\mu \circ v$ belongs to $M(\alpha)$, then, for arbitrary $\varepsilon>0$, there exists $\delta>0$ such that

$$
\limsup _{x \rightarrow \infty} \frac{\int_{0}^{\delta} t^{\alpha} \nu(d t) \int_{0}^{x / t} s^{\alpha} \mu(d s)}{\int_{0}^{x} t^{\alpha} \mu \circ v(d t)} \leq \varepsilon .
$$

PROOF. We can choose a positive constant $C$ such that $\int_{0}^{x} t^{\alpha} \mu(d t) / \int_{0}^{x} t^{\alpha} \mu$ 。 $v(d t)<C$ for large $x$ by Lemma 4.2. Let $V(x)=\int_{0}^{x} t^{\alpha} \mu \circ \nu(d t), U(x)=$ $\int_{0}^{\delta} t^{\alpha} \nu(d t) \int_{0}^{x / t} s^{\alpha} \mu(d s)$, where $\delta$ is a positive constant satisfying $C \int_{0}^{\delta} t^{\alpha} \nu(d t)<\varepsilon$. We have

$$
U(x) \leq C \int_{0}^{\delta} t^{\alpha} v(d t) \int_{0}^{x / t} s^{\alpha} \mu \circ v(d s) .
$$

On the other hand, by the representation theorem of s.v. function, we have $\int_{0}^{x} s^{\alpha} \mu$ 。 $v(d s)=c(x) \exp \left(\int_{B}^{x} \varepsilon(u) u^{-1} d u\right)$, where $\lim _{x \rightarrow \infty} c(x)=c(0<c<\infty)$ and $\lim _{u \rightarrow \infty} \varepsilon(u)=0$. Since

$$
\sup _{0<i \leq \delta} \int_{x}^{x / t} \frac{\varepsilon(u)-\alpha}{u} d u=\int_{x}^{x / \delta} \frac{\varepsilon(u)-\alpha}{u} d u
$$

for sufficiently large $x$, we get

$$
\lim _{x \rightarrow \infty} \sup _{0<t \leq \delta} \frac{\int_{0}^{x / t} s^{\alpha} \mu \circ v(d s)}{\int_{0}^{x} s^{\alpha} \mu \circ v(d s)}\left(\frac{t}{\delta}\right)^{\alpha}=1 .
$$

Hence

$$
\limsup _{x \rightarrow \infty} \int_{0}^{\delta} \frac{\int_{0}^{x / t} s^{\alpha} \mu \circ v(d s) t^{\alpha}}{V(x)} v(d t) \leq \int_{0}^{\delta} t^{\alpha} v(d t)
$$

By (4.2) and (4.3), $\lim \sup _{x \rightarrow \infty} U(x) / V(x) \leq C \int_{0}^{\delta} t^{\alpha} \nu(d t)<\varepsilon$. 
Using this lemma, we get the following propositions.

PROPOSITION 4.4. If $\mu \circ v$ is in $M(\alpha)$ and $v$ has finite $\alpha$ th moment, then $\mu$ belongs to $M(\alpha)$.

Proof. Let $U^{c}(x)=V(x)-U(x)=\int_{\delta}^{\infty} t^{\alpha} v(d t) \int_{0}^{x / t} s^{\alpha} \mu(d s)$ in the proof of Lemma 4.3. From this lemma, for $0<\varepsilon<1$, we can choose $\delta>0$ such that $\liminf _{x \rightarrow \infty} U^{c}(x) / V(x)>0$ and $v(\delta, \infty)>0$. It follows from Theorem $2.2(2)$ that $U^{c}(x)$ is s.v. If we choose a constant $B$ satisfying $\int_{\delta}^{B} t^{\alpha} \nu(d t)>0$, then

$$
\liminf _{x \rightarrow \infty} \frac{\int_{\delta}^{B} t^{\alpha} v(d t) \int_{0}^{x / t} s^{\alpha} \mu(d s)}{\int_{B}^{\infty} t^{\alpha} v(d t) \int_{0}^{x / t} s^{\alpha} \mu(d s)} \geq \frac{\int_{\delta}^{B} t^{\alpha} v(d t)}{\int_{B}^{\infty} t^{\alpha} v(d t)}>0 .
$$

By Theorem $2.2(2), \int_{\delta}^{B} \int_{0}^{x / t} s^{\alpha} \mu(d s) t^{\alpha} \nu(d t)$ is an s.v. component of $U^{c}(x)$. Noticing that $\delta>0$ and

$$
\int_{\delta}^{B} \int_{0}^{\delta x / t} s^{\alpha} \mu(d s) t^{\alpha} v(d t) \leq \int_{\delta}^{B} t^{\alpha} v(d t) \int_{0}^{x} t^{\alpha} \mu(d t) \leq \int_{\delta}^{B} t^{\alpha} \nu(d t) \int_{0}^{B x / t} t^{\alpha} \mu(d t),
$$

we get $\int_{0}^{x} t^{\alpha} \mu(d t)$ is s.v.

PROPOSITION 4.5. If $\mu \circ v$ belongs to $M(\alpha)$, then

$$
\limsup _{x \rightarrow \infty} \int_{0}^{x} t^{\alpha} \mu \circ v(d t) /\left(\int_{0}^{x} t^{\alpha} \mu(d t) \int_{0}^{x} t^{\alpha} v(d t)\right) \leq 1 .
$$

PROOF. By Lemma 4.3, for arbitrary $\varepsilon>0$, we can take $\delta>0$ such that $\limsup _{x \rightarrow \infty} \frac{\int_{0}^{\delta} t^{\alpha} v(d t) \int_{0}^{x / t} s^{\alpha} \mu(d s)}{\int_{0}^{x} t^{\alpha} \mu \circ v(d t)} \leq \varepsilon \quad$ and $\quad \limsup _{x \rightarrow \infty} \frac{\int_{0}^{\delta} t^{\alpha} \mu(d t) \int_{0}^{x / t} s^{\alpha} v(d s)}{\int_{0}^{x} t^{\alpha} \mu \circ v(d t)} \leq \varepsilon$.

Therefore we have

$$
\begin{gathered}
\limsup _{x \rightarrow \infty} \frac{\int_{0}^{x} t^{\alpha} \mu \circ v(d t)}{\int_{\delta}^{\infty} t^{\alpha} \nu(d t) \int_{\delta}^{x / t} s^{\alpha} \mu(d s)} \leq \frac{1}{1-2 \varepsilon}, \\
\limsup _{x \rightarrow \infty} \int_{0}^{x} t^{\alpha} \mu \circ v(d t) /\left(\int_{0}^{x} t^{\alpha} \mu(d t) \int_{0}^{x} t^{\alpha} \nu(d t)\right) \\
=\limsup _{x \rightarrow \infty} \int_{0}^{x} t^{\alpha} \mu \circ v(d t) /\left(\int_{0}^{x / \delta} t^{\alpha} \mu(d t) \int_{0}^{x / \delta} t^{\alpha} v(d t)\right) \\
\leq \limsup _{x \rightarrow \infty} \int_{0}^{x} t^{\alpha} \mu \circ v(d t) /\left(\int_{\delta}^{\infty} t^{\alpha} \mu(d t) \int_{\delta}^{x / t} s^{\alpha} v(d s)\right)
\end{gathered}
$$




$$
\leq \frac{1}{1-2 \varepsilon} \text {. }
$$

Letting $\varepsilon \rightarrow 0$, we have completed the proof.

The following proposition gives another condition for every factor to belong to $M(\alpha)$.

PROPOSITION 4.6. $\mu \circ v$ belongs to $S(\alpha)$ if and only if both $\mu$ and $v$ are in $S(\alpha)$. In this case,

$$
\lim _{x \rightarrow \infty} \int_{0}^{x} t^{\alpha} \mu \circ v(d t) /\left(\int_{0}^{x} t^{\alpha} \mu(d t) \int_{0}^{x} t^{\alpha} v(d t)\right)=1 .
$$

PROOF. Since $S(\alpha)$ is a subclass of $\boldsymbol{M}(\alpha)$ and $\boldsymbol{M}(\alpha)$ is closed under MS-convolution, $\mu \circ \nu$ is in $M(\alpha)$. Hence it follows from Proposition 4.5 and the assumption that

$$
\limsup _{x \rightarrow \infty} \int_{0}^{x^{2}} t^{\alpha} \mu \circ v(d t) /\left(\int_{0}^{x} t^{\alpha} \mu(d t) \int_{0}^{x} t^{\alpha} v(d t)\right) \leq 1 .
$$

Since

$$
\int_{0}^{x^{2}} t^{\alpha} \mu \circ v(d t) /\left(\int_{0}^{x} t^{\alpha} \mu(d t) \int_{0}^{x} t^{\alpha} v(d t)\right) \geq 1
$$

for any distributions $\mu$ and $\nu$ in $\boldsymbol{P}$, the left-hand side of (4.5) is not less than 1.

REMARK. Though (4.4) and (4.6) give the relation between the truncated moments of MS-convolution and the product of those of their factors, the asymptotic orders of the following three can be different from each other:

$$
\int_{0}^{x} t^{\alpha} \mu \circ v(d t), \quad \int_{0}^{x} t^{\alpha} \mu(d t) \int_{0}^{x} t^{\alpha} v(d t), \quad \int_{0}^{x^{2}} t^{\alpha} \mu \circ v(d t) .
$$

Hence if $\mu$ in $F(\alpha)$ satisfies $\lim _{x \rightarrow \infty} \int_{0}^{x} t^{\alpha} \mu(d t) / \log x=1$, then $\lim _{x \rightarrow \infty} \int_{0}^{x} t^{\alpha} \mu$ 。 $\mu(d t) / \log x=\infty$ by (4.6) (or Lemma 4.2). Therefore, $\mu \circ \mu \notin \boldsymbol{F}(\alpha)$ by Theorem 2.2 (4) and we see that $\boldsymbol{F}(\alpha)$ is not closed under MS-convolution.

The following theorem shows that Maller's conjecture is not true.

THEOREM 4.7. If $\mu$ is in $M(\alpha)$ with infinite $\alpha$ th moment and $v$ is in $C(\alpha)$, then $\mu \circ v$ belongs to $M(\alpha)$. 
Proof. Let $C$ be a constant such that $\int_{0}^{x^{2}} s^{\alpha} v(d s) / \int_{0}^{x} s^{\alpha} v(d s)<C$ for large $x$. In a similar way to the proof of Theorem 3.2,

$$
\frac{\int_{x}^{2 x} t^{\alpha} \mu \circ v(d t)}{\int_{0}^{x} t^{\alpha} \mu \circ v(d t)} \leq \sup _{t<\sqrt{2 x}} \frac{\int_{x / t}^{2 x / t} s^{\alpha} \mu(d s)}{\int_{0}^{x / t} s^{\alpha} \mu(d s)}+\frac{\int_{0}^{\sqrt{2 x}} t^{\alpha} \mu(d t) \int_{x / t}^{2 x / t} s^{\alpha} v(d s)}{\int_{0}^{x} t^{\alpha} \mu \circ v(d t)} .
$$

The first term tends to 0 as $x \rightarrow \infty$ by $\mu \in M(\alpha)$. Since $\int_{0}^{x} t^{\alpha} \mu \circ v(d t) \geq$ $\int_{0}^{\sqrt{x / 2}} t^{\alpha} \mu(d t) \int_{0}^{\sqrt{2 x}} t^{\alpha} v(d t)$, it is sufficient to prove that

$$
\lim _{x \rightarrow \infty} \frac{\int_{0}^{\sqrt{2 x}} t^{\alpha} \mu(d t) \int_{x / t}^{2 x / t} s^{\alpha} \nu(d s)}{\int_{0}^{\sqrt{x / 2}} t^{\alpha} \mu(d t) \int_{0}^{\sqrt{2 x}} t^{\alpha} \nu(d t)}=0
$$

We split the numerator into three parts and estimate each term:

$$
\begin{aligned}
& \int_{0}^{\sqrt{2 x}} t^{\alpha} \mu(d t) \int_{x / t}^{2 x / t} s^{\alpha} \nu(d s) \\
& =\int_{0}^{1} t^{\alpha} \mu(d t) \int_{x / t}^{2 x / t} s^{\alpha} \nu(d s)+\int_{1}^{\sqrt{2 x}} t^{\alpha} \mu(d t) \int_{x / t}^{2 x / t} s^{\alpha} \nu(d s) \\
& =\int_{2 x}^{\infty} s^{\alpha} \nu(d s) \int_{x / s}^{2 x / s} t^{\alpha} \mu(d t)+\int_{x}^{2 x} s^{\alpha} \nu(d s) \int_{x / s}^{1} t^{\alpha} \mu(d t) \\
& +\int_{1}^{\sqrt{2 x}} t^{\alpha} \mu(d t) \int_{x / t}^{2 x / t} s^{\alpha} \nu(d s) \\
& \int_{2 x}^{\infty} s^{\alpha} \nu(d s) \int_{x / s}^{2 x / s} t^{\alpha} \mu(d t)=\sum_{k=1}^{\infty} \int_{(2 x)^{2 k-1}}^{(2 x)^{2^{k}}} s^{\alpha} \nu(d s) \int_{x / s}^{2 x / s} t^{\alpha} \mu(d t) \\
& \leq \sum_{k=1}^{\infty} \int_{(2 x)^{2^{k-1}}}^{(2 x)^{2^{k}}} s^{\alpha} \mathcal{v}(d s) \int_{1 / 2(2 x)^{2^{k}-1}}^{1 /(2 x)^{2^{k-1}-1}} t^{\alpha} \mu(d t) \\
& \leq \sum_{k=1}^{\infty} C^{k} \int_{0}^{2 x} s^{\alpha} \nu(d s) \int_{1 / 2(2 x)^{2^{k-1}}}^{1 /(2 x)^{2^{k-1}-1}} t^{\alpha} \mu(d t) \\
& \leq \sum_{k=1}^{\infty} \frac{C^{k}}{(2 x)^{\left(2^{k-1}-1\right) \alpha}} \int_{0}^{2 x} s^{\alpha} v(d s) \\
& \int_{x}^{2 x} s^{\alpha} \nu(d s) \int_{x / s}^{1} t^{\alpha} \mu(d t) \leq \int_{0}^{1} t^{\alpha} \mu(d t) \int_{x}^{2 x} s^{\alpha} v(d s) \leq \int_{0}^{1} t^{\alpha} \mu(d t) \int_{0}^{2 x} s^{\alpha} \nu(d s) .
\end{aligned}
$$

The last term is the most important and estimated as follows. Define $n=n(x) \in N$ as $2^{n-1} \leq \sqrt{2 x}<2^{n}$. Then 


$$
\begin{aligned}
\int_{1}^{\sqrt{2 x}} t^{\alpha} \mu(d t) \int_{x / t}^{2 x / t} s^{\alpha} \nu(d s) & \leq \sum_{k=1}^{n} \int_{2^{k-1}}^{2^{k}} t^{\alpha} \mu(d t) \int_{x / t}^{2 x / t} s^{\alpha} v(d s) \\
& \leq \sum_{k=1}^{n} \int_{2^{k-1}}^{2^{k}} t^{\alpha} \mu(d t) \int_{x / 2^{k}}^{4 x / 2^{k}} s^{\alpha} v(d s) \\
& \leq \sup _{1 \leq i \leq n} \int_{2^{i-1}}^{2^{i}} t^{\alpha} \mu(d t) \sum_{k=1}^{n} \int_{x / 2^{k}}^{4 x / 2^{k}} s^{\alpha} v(d s) \\
& \leq 2 \sup _{1 \leq i \leq n} \int_{2^{i-1}}^{2^{i}} t^{\alpha} \mu(d t) \int_{x / 2^{n}}^{2 x} s^{\alpha} v(d s) \\
& \leq 2 \sup _{1 \leq i \leq n} \int_{2^{i-1}}^{2^{i}} t^{\alpha} \mu(d t) \int_{0}^{2 x} s^{\alpha} v(d s) .
\end{aligned}
$$

Thus we have

$$
\begin{aligned}
\int_{0}^{\sqrt{2 x}} & t^{\alpha} \mu(d t) \int_{x / t}^{2 x / t} s^{\alpha} \nu(d s) \\
& \leq\left(2 \sup _{1 \leq i \leq n} \int_{2^{i-1}}^{2^{i}} t^{\alpha} \mu(d t)+\sum_{k=1}^{\infty} \frac{C^{k}}{(2 x)^{\left(2^{k-1}-1\right) \alpha}}+\int_{0}^{1} t^{\alpha} \mu(d t)\right) \int_{0}^{2 x} s^{\alpha} v(d s) \\
& \leq C\left(2 \sup _{1 \leq i \leq n} \int_{2^{i}-1}^{2^{i}} t^{\alpha} \mu(d t)+\sum_{k=1}^{\infty} \frac{C^{k}}{(2 x)^{\left(2^{k-1}-1\right) \alpha}}+\int_{0}^{1} t^{\alpha} \mu(d t)\right) \int_{0}^{\sqrt{2 x}} s^{\alpha} v(d s) .
\end{aligned}
$$

Since $\mu$ belongs to $M(\alpha)$ and has infinite $\alpha$ th moment, we get $\lim _{x \rightarrow \infty} \sup _{1 \leq i \leq n}$ $\int_{2^{i-1}}^{2^{i}} t^{\alpha} \mu(d t) / \int_{0}^{\sqrt{x / 2}} t^{\alpha} \mu(d t)=0$. It is easy to show that $\lim _{x \rightarrow \infty} \sum_{k=1}^{\infty} C^{k} /(2 x)^{\left(2^{k-1}-1\right) \alpha}$ $=0$. Using these facts, we get (4.7).

REMARK. By Theorem 4.7, it can occur that $\mu \circ \nu$ belongs to $\boldsymbol{M}(\alpha)$ for $\mu \in \boldsymbol{M}(\alpha)$ and $v \notin \boldsymbol{M}(\alpha)$ even if $\lim _{x \rightarrow \infty} \int_{0}^{x} t^{\alpha} \mu(d t) / \int_{0}^{x} t^{\alpha} v(d t)=0$.

We construct a distribution in $\boldsymbol{C}(\alpha) \backslash \boldsymbol{M}(\alpha)$ to show that it is not empty.

EXAMPLE. Let $f(x)=\log x$ and $r=e$. Define a discrete probability measure $p$ as follows: $p\left(\left\{e^{e^{k}}\right\}\right)=c e^{-2 e^{k}+k}$, where $c$ is a normalized constant and $k=0,1, \ldots$ Then $V(x)$, the truncated second moment of $p$, is

$$
V(x)=c \sum_{k=0}^{n} e^{k}=c \frac{e^{n+1}-1}{e-1} \quad \text { for } \quad e^{e^{n}} \leq x<e^{e^{n+1}} .
$$


If $x<e^{e^{n+1}}$, then $x^{2}<e^{n^{n+2}}$. Therefore we have $V\left(x^{2}\right) / V(x) \leq\left(e^{n+2}-1\right) /\left(e^{n+1}-1\right)$. Thus we conclude that

$$
\limsup _{x \rightarrow \infty} \frac{V(2 x)}{V(x)}=\limsup _{x \rightarrow \infty} \frac{V\left(x^{2}\right)}{V(x)}=e .
$$

REMARK. It is still open whether there exists a distribution in $D_{2}$ that can be decomposed into two factors neither of which belongs to $D_{2}$.

We add a general result to this problem. We say that $v$ belongs to the domain of partial attraction of a distribution $\mu$ if, for i.i.d. random variables $X_{n}$ with disribution $\nu$, there is an increasing sequence $m_{n}$ of positive integers such that, for some constants $A_{n} \in \boldsymbol{R}^{1}$ and $B_{n}>0$, the distribution of $B_{n}^{-1} \sum_{k=1}^{m_{n}} X_{k}-A_{n}$ converges to $\mu$ as $n \rightarrow \infty$.

PROPOSITION 4.8. Every factor of a distribution in $D_{2}$ belongs to the domain of the partial attraction of Gaussian distribution.

PROOF. Since $\mu \circ v$ belongs to $D_{2}, \mu \circ v$ has finite absolute $\alpha$ th moment for every $\alpha \in(0,2)$, which is equivalent to that both $\mu$ and $\nu$ have finite absolute $\alpha$ th moments for each $\alpha \in(0,2)$. Maller [4] shows that this implies that both $\mu$ and $v$ belong to the domain of partial attraction of Gaussian distribution.

\section{Acknowledgement}

The author would like to thank Professor K. Sato, as well as the referee, for their valuable comments on earlier imperfect versions of this paper.

\section{References}

[1] N. M. Bingham, C. M. Goldie and J. L. Teugels, Regular variation, Encyclopedia Math. Appl. (Cambridge University Press, Cambridge, 1987).

[2] W. Feller, An introduction to probability theory and its applications, volume II, second edition (Willey, New York, 1971).

[3] B. V. Gnedenko and A. N. Kolmogorov, Limit distributions for sums of independent random variables, second edition (Addison Wesley, Cambridge, 1968).

[4] R. A. Maller, 'A note on domain of partial attraction', Ann. Probab. 3 (1980), 576-583.

[5] —, 'A theorem on products of random variables, with application to regression', Austral. $J$. Statist. 23 (1981), 177-185.

[6] E. Seneta, Regularly varying functions, Lecture Notes in Math. 508 (Springer, Berlin, 1976).

[7] T. Shimura, 'Decomposition of non-decreasing slowly varying functions and the domain of attraction of Gaussian distributions', J. Math. Soc. Japan 43 (1991), 775-793. 
[8] — - 'Decomposition of probability measures related to monotone regularly varying functions', Nagoya Math. J. 135 (1994), 87-111.

The Institute of Statistical Mathematics

4-6-7 Minami-Azabu

Minato-ku

Tokyo 106

Japan

e-mail: shimura@ism.ac.jp 